\title{
An intervention program to reduce the number of hospitalizations of elderly patients in a primary care clinic Roni Peleg*1,2, Yan Press ${ }^{1,2}$, Maya Asher1, Tatyana Pugachev1,2, Hadas Glicensztain ${ }^{1}$, Mila Lederman ${ }^{1}$ and Aya Biderman ${ }^{1,2}$
}

\author{
Address: ${ }^{1}$ Clalit Health Services, Southern District, Israel and ${ }^{2}$ Department of Family Medicine, Faculty of the Health Sciences, Ben-Gurion \\ University of the Negev, Beer-Sheva, Israel \\ Email: Roni Peleg* - pelegr@bgumail.bgu.ac.il; Yan Press - yanp@zahav.net.il; Maya Asher - mayas@clalit.org.il; \\ Tatyana Pugachev - pelegr@bgu.ac.il; Hadas Glicensztain - pelegr@clalit.org.il; Mila Lederman - ronipel@gmail.com; \\ Aya Biderman - sbider@netvision.net.il \\ * Corresponding author
}

Published: 6 February 2008

BMC Health Services Research 2008, 8:36 doi:10.1186/1472-6963-8-36

Received: 18 August 2007

Accepted: 6 February 2008

This article is available from: http://www.biomedcentral.com/1472-6963/8/36

C 2008 Peleg et al; licensee BioMed Central Ltd.

This is an Open Access article distributed under the terms of the Creative Commons Attribution License (http://creativecommons.org/licenses/by/2.0), which permits unrestricted use, distribution, and reproduction in any medium, provided the original work is properly cited.

\begin{abstract}
Background: The elderly population consumes a large share of medical resources in the western world. A significant portion of the expense is related to hospitalizations.

Objectives: To evaluate an intervention program designed to reduce the number of hospitalization of elderly patients by a more optimal allocation of resources in primary care.

Methods: A multidimensional intervention program was conducted that included the reengineering of existing work processes with a focus on the management of patient problems, improving communication with outside agencies, and the establishment of a system to monitor quality of healthcare parameters. Data on the number of hospitalizations and their cost were compared before and after implementation of the intervention program.
\end{abstract}

Results: As a result of the intervention the mean expenditure per elderly patient was reduced by $22.5 \%$. The adjusted number of hospitalizations/I,000 declined from I5.I to 10.7 (29.3\%). The number of adjusted hospitalization days dropped from 132 to $82(37.9 \%)$ and the mean hospitalization stay declined from 8.2 to 6.7 days (17.9\%). The adjusted hospitalization cost $(\$ / 1,000$ patients) dropped from $\$ 32,574$ to $\$ 18,624$ (42.8\%). The overall clinic expense, for all age groups, dropped by $9.9 \%$.

Conclusion: Implementation of the intervention program in a single primary care clinic led to a reduction in hospitalizations for the elderly patient population and to a more optimal allocation of healthcare resources.

\section{Background}

The rise in life expectancy in the western world has led to a significant increase in the number of individuals over the age of 65 years. The percentage of elderly has reached
$19.1 \%$ in Italy, ranked the world's oldest country, followed by Japan at 19\% [1].

In Israel at the end of 2002 there were approximately 655,000 elderly individuals, aged 65 and above, compris- 
ing just under $10 \%$ of the population [2]. Life expectancy in Israel is among the highest in the world and is currently 81.2 years for women and 77.3 years for men. While the elderly population increased in absolute terms by $48 \%$ in the years 1990 to 2002, the number of those older than 80 years grew by $69 \%$ [2]. This population group is particularly susceptible to cognitive and functional impairment [3-5], depression [6,7], falls [8], and visual [9] or hearing impairment [10]. The elderly population is the largest consumer of healthcare resources in the western world. A large part of these expenses stems from the hospitalization of elderly patients in medical centers. They are also more likely to have inadequate financial stability and to be unaware of available social support services [11]. Frequently, such conditions are overlooked by the primary care physician $[12,13]$.

Improvement in the care of elderly patients and more optimal use of healthcare resources, including hospitalizations, represents an important challenge for healthcare services in the world.

In the study clinic the adjusted per patient cost is high compared to regional and national averages with an increase in costs over the years. The primary cause of this disparity is the cost of hospitalization, primarily among patients 65 years of age and above.

The objective of the present study was to evaluate the results of an intervention program designed to improve care for elderly patients, to reduce hospitalizations, and to generate a more optimal use of healthcare resources.

\section{Methods \\ Setting}

The compulsory national health insurance system, implemented in Israel in 1995, provides healthcare to the entire population through non-profit health maintenance organizations (sick funds). Four sick funds provide healthcare services to the Israeli population based on the patient-oriented medicine model, which entails the provision of treatment by a primary care physician and a nurse working as a team, and specialist consultations as required. The present study was conducted within the framework of a quality improvement program in the Clalit Health Services (CHS) for the southern district of Israel. CHS is Israel's largest sick fund, serving about $53 \%$ of the population.

The intervention program and its evaluation were conducted in a primary care clinic in Beer-Sheva, the capitol city of the southern district. The clinic, which is located about 5 minutes by foot from the central city hospital (Soroka Medical Center), provides primary healthcare services for 4,620 registered clients of all ages. The number of insured elderly patients 65 years old or above is 808 , $17.5 \%$ of the clinic's registry. The clinic is located in one of the city's older neighborhoods. The population is generally of low socioeconomic status with $22.6 \%$ receiving support from Israel's Social Security Institute compared to $13.0 \%$ of the registered clients of CHS throughout the country. The clinic staff includes three family physicians and one pediatrician, two nurses, an administrative director, and a part-time administrator whose task is to promote facilitate the independent functioning of the clinic. All medical records are computerized and statistical and economic data are available, including quality of care indicators. Patients mostly have scheduled appointments with the doctors at ten minutes intervals. The clinic has an annual budget for which it is independently responsible.

\section{Baseline data prior to implementation of the intervention program - patients 65 years or above}

During the course of 2004 the mean expenditure for patients 65 years of older was $\$ 2,273$, compared to a mean of $\$ 1,840$ for the region (a difference of $21.1 \%$ ). The adjusted mean number of hospitalizations per 1,000 patients was 15.1 in the clinic compared to 11.1 for the region (a difference of $35.7 \%$ ). The adjusted mean number of hospitalization days per 1,000 was $132 \mathrm{com}$ pared to 82 for the region (59\%), and the adjusted mean cost of hospitalization per 1,000 in the clinic was $\$ 32,574$ compared to $\$ 21,794$ for the region $(49.5 \%)$. The mean duration of hospitalization for the clinic was 8.2 days compared to 7.2 for the region. Data on hospitalizations for elderly patients appear in Table 1.

Table I: Hospitalizations for patients 65 and older in 2004 ( I USD = 4.45 NIS).

\begin{tabular}{|c|c|c|c|}
\hline & Southern district & Study clinic & Difference (\%) \\
\hline Cost per registered patient $(\$)$ & $\mathrm{I}, 840$ & 2,227 & $387(21.1)$ \\
\hline Adjusted hospitalizations/I,000 patients & II.I & I5. I & $3.97(35.7)$ \\
\hline Adjusted duration of hospitalization (days/I,000) & 83 & 132 & 49 (59) \\
\hline Adjusted hospitalization cost $(\$ / I, 000)$ & 21,794 & 32,574 & $10,780(49.5)$ \\
\hline Mean duration of hospitalization (days) & 7.2 & 8.2 & I (I3.8) \\
\hline
\end{tabular}




\section{Definition of the problem}

There is a significantly higher cost of hospitalization for elderly patients between the study clinic and the regional average.

\section{Analysis of the causes of this problem}

As a first step the clinic staff analyzed the causes of the high costs of hospitalization.

\section{The medical system problem}

1. Lack of a modern monitoring system to follow hospitalized patients and patients who are bedridden or have particularly severe disease.

2. Lack of an internal control system to maintain communication with hospitalized patients and their families.

3. Lack of a mechanism to follow patients after discharge from the hospital and guarantee continuity of care.

4. A less than optimal medical record of patients with newly diagnosed chronic disease and of patients with existing chronic disease.

5. The absence of a social worker and a dietician in the clinic staff.

6. Insufficient health promotion and disease prevention activity on the part of the clinic staff and patients.

7. There is insufficient contact and only partial collaboration with the hospital wards, the emergency room, the hospitalization monitoring unit, and the home hospice unit.

\section{Patients and environment}

1. $17.5 \%$ of the clinic population are elderly, i.e., 65 years of age or older.

2. The socioeconomic status is low with $22.6 \%$ supported by the Israel Social Security Institute.

3. Low compliance rate with treatment regimen and follow-up visits.

4. The patients are unaware of other healthcare service options in the community.

5. The clinic is geographically situated close to the hospital.

\section{The intervention program}

In light of the definition of the problem and the analysis of its causes we chose solutions that were designed to reduce the number of hospitalizations among elderly patients. The basic assumption that underpinned the choice of solutions was that improved quality of care will lead to optimization of the utilization of healthcare resources and to reduced costs. The clinic staff chose to intervene in those areas in which intervention could lead to change. For example, it would be unrealistic to intervene in factors such as the geographical location of the clinic or its socioeconomic status. However, resources could be allocated for a social worker and a dietician, and the level of cooperation with outside agencies could be enhanced. The program was developed in an ongoing manner throughout the year 2005.

Improvement of existing work processes and development of new processes

1. Identification of patients with multiple hospitalizations, patients with chronic diseases who were not well controlled, and patients who were hospitalized more than twice over the course of the previous year. This list of patients was disseminated among the clinic physicians who initiated appointments with them and coordinated the appointments with the clinic nursing staff. An intervention program was designed for each patient, in accordance with his or her specific needs.

\section{A geriatrics specialist liaison service in the clinic:}

a. The geriatrician visited the clinic once a week, reviewed the computerized patients' charts and appended electronic reminder notes regarding evaluation and treatment of elderly patients.

b. The geriatrician conducted joint meetings with physicians and patients, as a liaison.

c. Provide patients with formal internal clinic referral forms and set an appointment for them with their family physician to guarantee continuity of care for specific health problems that were identified in the joint meeting.

d. The geriatrician gave relevant lectures to the clinic staff and conducted staff meetings on issues such as prevention of falls in the elderly, polypharmacy, and the use of benzodiazepines among elderly patients.

3. Establishment of a clinic hospitalization monitor system. The team included the medical, nursing and administrative directors of the clinic. Hospital reports were printed out from the computer database and reviewed on a daily basis.

4. Health promotion and disease prevention were emphasized as critical aspects of primary care. The clinic staff prepared presentations on subjects such as the principles of 
disease prevention, disease prevention in adults, and early detection of colorectal and breast cancer.

\section{Improvement of communication and management of patient} problems

1. Individual training regarding the care of emergency cases.

2. Publication of a short newsletter on referrals to the emergency room.

3. Provision of personal telephone numbers of the medical staff to a selected group of patients (at the discretion and judgment of the treating physician).

4. The addition of a part-time social worker to help with the provision of social support to patients and the clinic staff.

5. The addition of a dietician, five hour per week, to counsel patient with metabolic and other disorders.

Improvement of communication with outside agencies

1. A meeting of the clinic staff with the staff of the internal medicine ward in which the clinic patients are regularly hospitalized. At the meeting the two staffs jointly discussed ways to improve hospitalization and discharge procedures as well as means to improve communication between the hospital and the clinic staff.

2. A meeting of the entire clinic staff with the emergency room staff in which the two staffs discussed issues such as improving referrals to the emergency room and improved "real-time" communication between the teams.

3. Strengthened ties with the hospital's hospitalizationmonitoring unit and the development of a "service contract". The treating physician completed a structured form for every patient who remained in the hospital for more than five days. The form included the clinic physician's position as to the need for continued hospitalization and the feasibility of alternative care in the community. The clinic monitoring team transferred these recommendations to the hospital's hospitalization-monitoring team, which assessed each clinic recommendation on an individual basis. In some cases this process was also applied to patients who were hospitalized for less than five days.

4. Strengthening of ties with CHS's southern region home hospice team. Terminally ill patients who were considered suited for this type of care were provided optimal palliative care at home.
5. Direct collaboration with the person responsible for reducing patient waiting lists for outpatient consultations and elective hospitalizations.

Establishment in the clinic of a monitoring system to increase awareness of the importance of improved quality of care and optimal utilization of resources

1. Data on the quality indices and the use of resources were presented at staff meetings four times during the year.

2. The clinic economist held meetings with each clinic physician at which time they discussed data on the individual quality indices and use of resources in the physician's unit. These meetings were held every six months. The aim of the meetings was to apprise the physicians of what was taking place in their individual units.

3. The clinic's directorate conducted ongoing quality control assessments on quality of healthcare parameters in the clinic and the use of healthcare resources.

The medical and nursing staffs were encouraged to conduct regular visits with hospitalized and home-ridden patients and to be in touch with their families.

The clinic staff participated in the regional directorate's forum for "high-cost clinics". Reports on ongoing processes were presented at these meetings and there was an exchange of views and ideas among the staffs of the various clinics. Five forum meetings were held in 2005.

During the same time period the regional directorate initiated a program for all the clinics of the region in which all patients over the age of 65 were "mapped" and evaluated on the basis of a structured plan.

To evaluate the effectiveness of the intervention we chose the following outcomes:

1. Quality measurement outcomes such as the number of hospitalizations, the number of eye examinations for diabetic patients, and parameters of diabetes control.

2. Economic outcomes such as overall cost, cost of hospitalizations, cost of ambulatory treatments, and cost of ancillary tests.

\section{Results}

As a result of the intervention program the actual mean overall expenditure per individual registered in the clinic decreased to $\$ 1,727$ compared to $\$ 1,848$ for the region, a drop of $22.5 \%$ in terms of the corresponding costs for the clinic in 2004. The adjusted hospitalization rate per 1,000 dropped by $29.3 \%$, the adjusted number of hospitaliza- 
tion days per 1,000 patients dropped by $37.9 \%$, the mean adjusted cost of hospitalization dropped by $42.8 \%$, and the mean hospital stay dropped to 6.7 days compared to 7.1 for the region, a drop of $17.9 \%$ compared to 2004 for our clinic.

Table 2 presents details on hospitalization for clinic patients 65 years of age and older for 2005 compared to 2004, and in comparison with data from the region.

\section{Medical quality indices}

Any intervention aimed at reducing costs must ensure that the quality of care is not impaired. Two hundred eighty five elderly patients received Pneumovax vaccinations in 2005 compared to 74 in 2004, an increase of $385 \%$. Improvement was also seen in indices related to diabetes mellitus: the number of well-controlled diabetes patients $\left(\mathrm{HbA}_{1} \mathrm{C}<7\right)$, the number of diabetic patients tested for microalbuminuria, and the overall follow-up.

In contrast, there was a decrease in the amount of drugs used to reduce serum lipids and in care for microalbumin. Quality-of-care data are presented in Table 3.

\section{The effect of the intervention program on the overall use of healthcare resources}

During 2005 there was an overall reduction of $9.9 \%$ in total clinic expenditure compared to 2004, despite an increase of $2.4 \%$ in the clinic population over the course of that year. The major cost reductions were hospitalization for acute illness (11.9\%) and prolonged illness $(63.0 \%)$. There was a corresponding increase in the cost of ambulatory care such as hospital day care and imaging procedures. Table 4 presents a comparison of total costs between 2004 and 2005 for all clinic age groups.

\section{Discussion}

The clinic staff decided to conduct and evaluate an intervention program to reduce hospitalizations among elderly patients in the clinic because of the large effect of this parameter on costs and the utilization of the clinic resources. The program is unique in that it was developed in a structured format by the clinic staff itself. It received the support of the regional directorate as manifested in the provision of new manpower resources such as a geriatric consultant, a social worker, and a dietician. The design and development of the intervention program was an ongoing process that continued throughout the year. The focus was global rather than on a specific medical problem.

We conducted a multidimensional intervention program that included the re-engineering of existing work processes with a focus on the management of patient problems, improving communication with outside agencies and the establishment of a system to monitor healthcare parameters. Organization change in the primary care setting has been shown to be justified in previous reports $[14,15]$ including care management for low- income seniors [16].

The success of the intervention is reflected in the reduction of the number of hospitalizations, the number of hospitalization days, the cost of hospitalization, the mean duration of hospitalization, and the overall clinic expenditure. The largest reduction in costs was for patients with prolonged hospitalizations (rehabilitation and complex nursing care). In a single clinic intervention it is possible that a small number of patients could have a significant effect on the results.

Intervention programs for the prevention of disease and the reduction of hospitalizations from the community are usually successful. An intervention program for secondary prevention of ischemic heart disease implemented by a nursing staff was shown to be cost-effective in terms of lives saved [17]. The planning of hospitalization, communication and education of patients led to earlier discharge without apparent increased morbidity in one program [18], and a similar program aimed at reducing hospitalizations for chest pain led to a reduction in hospitalization costs [19]. Support by the medical team for elderly patients in the community and cooperation with the hospital medical staff can bring about a significant improvement in hospital days, savings in expenses, and can result in better patient outcome $[19,20]$.

Table 2: Hospitalizations for patients 65 and older in 2005 (I USD = 4.45 NIS).

\begin{tabular}{|c|c|c|c|c|c|}
\hline & Southern district & Study clinic & Difference & $\begin{array}{l}\text { Compared to } \\
\text { region (\%) }\end{array}$ & $\begin{array}{l}\text { Compared to study } \\
\text { clinic in } 2004(\%)\end{array}$ \\
\hline Cost per registered patient $(\$)$ & 1,849 & $\mathrm{I}, 727$ & 122 & --6.6 & --22.5 \\
\hline Adjusted hospitalizations/I,000 patients & 10.3 & 10.7 & 0.36 & 3.5 & --29.3 \\
\hline Adjusted duration of hospitalization (days/I,000 patients) & 81 & 82 & I & 1.2 & --37.9 \\
\hline Adjusted hospitalization cost ( $\$ / 1,000$ patients) & 19,1756 & 18,624 & 550 & --2.9 & --42.8 \\
\hline Mean duration of hospitalization (days) & 7.1 & 6.7 & --0.4 & --5.5 & --17.9 \\
\hline
\end{tabular}


Table 3: Comparison of medical quality indices between 20042005 (\%).

\begin{tabular}{lccc}
\hline & 2004 & 2005 & Difference \\
\hline Diabetics - eye examination & 72.7 & 77.5 & 4.8 \\
Diabetics - HbA ${ }_{1} \mathrm{C}$ measured & 90.2 & 89.8 & --0.4 \\
Diabetics - HbA, $\mathrm{C} \leq 7$ & 41.8 & 54.2 & 12.4 \\
Diabetics - HbA ${ }_{1} \mathrm{C}$ moderately elevated & 44.2 & 37.5 & --6.7 \\
Diabetics - HbA, $\mathrm{C} \geq 9$ & 13.9 & 8.3 & --5.6 \\
Diabetics - LDL measured & 87.4 & 87.2 & --0.2 \\
Diabetics - LDL $\leq 100$ & 55.6 & 50.3 & --5.3 \\
Diabetics - LDL moderately elevated & 27.5 & 31.9 & 4.4 \\
Diabetics - LDL > I30 & 16.9 & 17.8 & 0.9 \\
Microalbumin measured & 75.4 & 81.3 & 5.9 \\
Microalbumin treated regularly & 76.5 & 72.0 & --4.5 \\
Medication for dyslipidemia & 44.4 & 39.4 & --5.0 \\
\hline
\end{tabular}

Our intervention program led to an increase of $385 \%$ in Pneumovax vaccinations in the elderly. Communityacquired pneumonia is an increasingly common disease among elderly patients [21]. Early discharge from the hospital combined with support by the medical care system in the community can reduce hospital days and nosocomial infections [22]. The achievement of the intervention program may have contributed to the reduction in illness in addition to the reduction in the number of days that elderly patients spent in the hospital. Improvement could be seen in other parameters of quality that were chosen as measures of the intervention's results, for example eye examinations, HbA1c levels, and diabetes control in general (Table 3). A decrease was also seen in mortality, from 14 patients in 2004 to nine patients in 2005.

Focusing on the elderly age group in our intervention program led, in the final analysis, to a positive change in the total clinic costs. There was a significant drop in the cost of hospitalization and a corresponding increase in the use of ambulatory medical resources. These changes reflect a more optimal use of healthcare resources.
Although the development of the program and its integration into clinic work required time and effort on the part of the entire staff in the course of an intense work schedule, its implementation brought other positive developments. The entire clinic staff reported an improvement in the quality of healthcare, including improved communication with patients, families, and medical agents outside the clinic. An example of the latter is the direct communication with the regional coordinator responsible for reducing waiting times, which led to quicker treatment for the patients and improved satisfaction for all sides.

The clinic staff felt that it's work was more efficient, that relations between staff members improved, and that it was better equipped to identify and solve problems at the clinic level in an independent manner.

There are several limits to the evaluation of this intervention program. Case studies can teach a lot about processes, but their outcomes may not be readily generalizable. The number of patients in the clinic is relatively small so that a change relating to a few patients could have a significant effect on the results.

There is no way of determining the specific contribution of each of the program's elements to the final outcome, nor can we isolate the effect of specific diseases such as ischemic heart disease and pneumonia on the final results.

We decided to evaluate the intervention program in a single clinic so as to gain information about its potential benefit at the single clinic level. Other factors, e.g., a particularly severe winter in the study year or mass media reporting of bird flu, could have affected referral rates to the emergency room or even the decision as to whether to hospitalize a particular patient.

There are differences throughout the world in terms of provision of healthcare services and culture-based atti-

Table 4: Comparison of major expenses for the entire study clinic population between 2004 and 2005 in USD (I USD = 4.45 NIS).

\begin{tabular}{lccc}
\hline \multicolumn{1}{c}{ Expense } & 2004 & 2005 & Difference (\%) \\
\hline General hospitalization & $1,121,910$ & 988,407 & $--133,503(--11.9)$ \\
Elective hospitalization & 495,218 & 476,412 & $-18,806(--3.8)$ \\
Ambulatory costs & 315,169 & 368,761 & $53,592(17.0)$ \\
Imaging procedures & 141,423 & 145,767 & $4,345(3.1)$ \\
Emergency room visits & 128,497 & 138,765 & $10,268(8.0)$ \\
Hospital day care & 59,157 & 65,028 & $5,871(9.9)$ \\
Prolonged illness (nursing care) & 266,785 & 98,817 & $--167,968(--63.0)$ \\
Medications & 207,759 & 183,321 & $--24,438(--11.8)$ \\
Disposables & 21,819 & 19,129 & $--2,690(--12.3)$ \\
Different patient payments & $--43,618$ & $--39,620$ & $3,998(9.2)$ \\
All expenses & $2,714,119$ & $2,444,787$ & $--269,332(--9.9)$
\end{tabular}


tudes to healthcare. Our intervention program was designed and implemented in accordance with local needs, so we cannot be certain of their generalizability to other countries and healthcare systems. Nevertheless, we hope that our experience will aid others in meeting the challenge of improving healthcare for the elderly while attaining a more optimal allocation of healthcare resources and improving quality of care.

\section{Conclusion}

We conclude that implementation of the intervention program in a single primary care clinic led to a reduction in hospitalizations for the elderly patient population and to a more optimal allocation of healthcare resources.

\section{Competing interests}

The author(s) declare that they have no competing interests.

\section{Authors' contributions}

RP initiated and coordinated the study and wrote the paper. YP was the geriatric consultant for the study and participated in writing the paper. MA headed the administration of the research project and liaison with the hospital staff. TP was a member of the project's lead team. HG served as the assistant study coordinator and held responsibility for data handling. ML was the nursing care coordinator for study and was responsibility for discharge of study patients from hospital. $\mathrm{AB}$ served as the external consultant for the research project and participated in writing the paper.

\section{References}

I. Kinsella K, Phillips DR: The challenge of success. Washington, DC: Population Reference Bureau; 2005.

2. Brodsky J, Schnoor Y, Be'er S: The elderly in Israel-Statistical Abstract. In Mashav-planning for the elderly: a national database Jerusalem, Israel: Myers-JDC-Brookdale Institute and Eshel; 2003.

3. Hebert R, Dion MH: Efficacy of in-home screening for risk of functional decline. Age Ageing 200I, 30:265-266.

4. Hebert R: Functional decline in old age. CMAJ 1997, I 57: 1037-1045.

5. Walaszek A, Snowden M: Repeat cognitive screening of initially normal, older primary-care patients. $A m ~ J$ Geriatr Psychiatry 2004, I 2(5):545-546.

6. Mulsant BH, Ganguli M: Epidemiology and diagnosis of depression in late life. J Clin Psychiatr 1999, 60(Suppl 20):9-15.

7. Ganguli M, Hendrie HC: Screening for cognitive impairment and depression in ethnically diverse older populations. Alzheimer Dis Assoc Disord 2005, 19:275-278.

8. Lin JT, Lane JM: Falls in the elderly population. Phys Med Rehabil Clin N Am 2005, 16:109-128.

9. Cacciatore F, Abete P, Maggi S, Luchetti G, Calabrese C, Viati L, Leosco D, Ferrara N, Vitale DF, Rengo F: Disability and 6-year mortality in elderly population. Role of visual impairment. Aging Clin Exp Res 2004, 16:382-388.

10. Green CA, Pope CR: Effects of hearing impairment on use of health services among the elderly. I Aging Health 200I, 13:315-328.

II. Berkman B, Shearer S, Simmons WJ, White M, Robinson M, Sampson $S$, Holmes W, Allison D, Thomson JA: Ambulatory elderly patients of primary care physicians: functional, psychosocial and environmental predictors of need for social work care management. Soc Work Health Care 1996, 22: I-20.
12. Calkins DR, Rubenstein LV, Cleary PD, Davies AR, Jette AM, Fink A, Kosecoff J, Young RT, Brook RH, Delbanco TL: Failure of physicians to recognize functional disability in ambulatory patients. Ann Intern Med I99/, I | 4:45I-454.

13. Maly RC, Hirsch SH, Reuben DB: The performance of simple instruments in detecting geriatric conditions and selecting community-dwelling older people for geriatric assessment. Age Ageing 1997, 26:223-231.

14. Iliffe S, Lenihan P, Wallace P, Drennan V, Blanchard M, Harris A: Applying community-oriented primary care methods in British general practice: a case study. Br J Gen Pract 2002, 52:646-65।.

15. Mclnnes E, Mira M, Atkin N, Kennedy P, Cullen J: Can GP input into discharge planning result in better outcomes for the frail aged: results from a randomized controlled trial. Fam Pract 1999, 16:289-293.

16. Counsell SR, Callahan CM, Clark DO, Tu W, Buttar AB, Stump TE, Ricketts GD: Geriatric care management for low-income seniors: a randomized controlled trial. JAMA 2007, 298:2623-2633.

17. Raftery JP, Yao GL, Murchie P, Campbell NC, Ritchie LD: Cost effectiveness of nurse led secondary prevention clinics for coronary heart disease in primary care: follow up of a randomised controlled trial. BMJ 2005, 330:707.

18. Engelman RM: Mechanisms to reduce hospital stays. Ann Thorac Surg 1996, 62:1890-1891.

19. Weingarten SR, Riedinger MS, Conner L, Lee TH, Hoffman I, Johnson $B$, Ellrodt AG: Practice guidelines and reminders to reduce duration of hospital stay for patients with chest pain. An interventional trial. Ann Intern Med 1994, I 20:257-263.

20. Schraeder C, Shelton P, Sager M: The effects of a collaborative model of primary care on the mortality and hospital use of community-dwelling older adults. J Gerontol A Biol Sci Med Sci 200I, 56:MI06-II2.

21. Fry AM, Shay DK, Holman RC, Curns AT, Anderson LJ: Trends in hospitalizations for pneumonia among persons aged 65 years or older in the United States, 1988-2002. JAMA 2005, 294:27| 2-27| 9

22. Rhew DC, Weingarten SR: Achieving a safe and early discharge for patients with community-acquired pneumonia. Med Clin North Am 200I, 85: I 427-I440.

\section{Pre-publication history}

The pre-publication history for this paper can be accessed here:

http://www.biomedcentral.com/1472-6963/8/36/prepub

Publish with Biomed Central and every
scientist can read your work free of charge
"BioMed Central will be the most significant development for
disseminating the results of biomedical research in our lifetime. "
Sir Paul Nurse, Cancer Research UK
Your research papers will be:
• available free of charge to the entire biomedical community
• peer reviewed and published immediately upon acceptance
• cited in PubMed and archived on PubMed Central
• yours - you keep the copyright
Submit your manuscript here:
http://www.biomedcentral.com/info/publishing_adv.asp

